Naomi Matsumoto - Shigehiko Tamura - Ann Moser

Hugo W. Moser • Nancy Braverman • Yasuyuki Suzuki

Nobuyuki Shimozawa - Naomi Kondo • Yukio Fujiki

\title{
The peroxin Pex6p gene is impaired in peroxisomal biogenesis disorders of complementation group 6
}

Received: December 25, 2000 / Accepted: February 5, 2001

\begin{abstract}
Human genetic peroxisomal biogenesis disorders (PBDs), such as Zellweger syndrome, comprise 13 different complementation groups (CGs). Eleven peroxin genes, termed PEXs, responsible for PBDs have been identified, whereas pathogenic genes for PBDs of 2CGs, CG-A (the same CG as CG8 in the United States and Europe) and CG6, remained unidentified. We herein provide several lines of novel evidence indicating that PEX6, the pathogenic gene for CG4, is impaired in PBD of CG6. Expression of $P E X 6$ restored peroxisome assembly in fibroblasts from a CG6 PBD patient. This patient was a compound heterozygote for PEX6 gene alleles. Accordingly, by merging CG6 with CG4, human PBDs are now classified into 12CGs.
\end{abstract}

Key words Peroxisomal biogenesis disorders - Complementation groups $\cdot$ Peroxin $\cdot$ PEX6 $\cdot$ AAA ATPase $\cdot$ Protein import

\section{Introduction}

Human fatal genetic peroxisomal biogenesis disorders (PBDs; MIM 601539) include Zellweger syndrome (ZS; MIM 214100), neonatal adrenoleukodystrophy (NALD;

N. Matsumoto $\cdot$ S. Tamura $\cdot$ Y. Fujiki $(\bowtie)$

Department of Biology, Faculty of Sciences, Kyushu University

Graduate School, 6-10-1 Hakozaki, Higashi-ku, Fukuoka 812-8581,

Japan

Tel. +81-92-642-2635; Fax +81-92-642-4214

e-mail: yfujiscb@mbox.nc.kyushu-u.ac.jp

N. Matsumoto $\cdot$ Y. Fujiki

CREST, Japan Science and Technology Corporation, Tokyo, Japan

A. Moser · H.W. Moser

Department of Neurology and Pediatrics, Kennedy-Krieger Institute, Johns Hopkins University, Baltimore, MD, USA

N. Braverman

Department of Pediatrics, Johns Hopkins University, Baltimore, MD, USA

Y. Suzuki $\cdot$ N. Shimozawa $\cdot$ N. Kondo

Department of Pediatrics, Gifu University School of Medicine, Gifu, Japan
MIM 202370), infantile Refsum disease (IRD; MIM 266510), and rhizomelic chondrodysplasia punctata (RCDP; MIM 215100) (Lazarow and Moser 1995). Genetic heterogeneity, comprising 16 different complementation groups (CGs), has been identified in mammals, including 13CGs of PBDs and 3 distinct CGs of mutant Chinese hamster ovary $(\mathrm{CHO})$ cell lines, as determined by cellfusion and/or peroxin gene (PEX) transfection, using fibroblasts from patients with PBDs and peroxisome-deficient CHO cell mutants (Ghaedi et al. 1999; Fujiki 2000) (see Table 1). The primary cause responsible for the peroxisome deficiency in the 13CGs of PBDs is a failure in peroxisome biogenesis (Fujiki 2000; Gould and Valle 2000). Eleven $P E X$ genes involved in peroxisome biogenesis - $P E X 1$, PEX2, PEX3, PEX5, PEX6, PEX7, PEX10, PEX12, $P E X 13, P E X 16$, and $P E X 19$ - have been identified as the genetic-related cause responsible for PBD groups $\mathrm{E}$ in Japan (CG1 in the United States and Europe), F (CG10), G (CG12), CG2, C (CG4), R (CG11), B (CG7), CG3, $\mathrm{H}$ (CG13), D (CG9), and J (CG14), respectively (Fujiki 2000; Gould and Valle 2000; Ghaedi et al. 2000; Shimozawa et al. 2000; Muntau et al. 2000; South et al. 2000). Thus, pathogenic genes responsible for two CGs, CG-A (CG8) and CG6, remain to be isolated (Fujiki 2000; Ghaedi et al. 2000).

The fibroblast cell line from a CG6 patient (PBD6-01) with NALD (patient 6 in the report of Kelly et al. (1986)) was difficult to study because of poor growth. PBD6-01 cells were previously shown to complement CG1 cells in cellfusion analysis (Moser et al. 1995). In regard to complementation with CG4 cell lines, it was concluded that CG6 was distinct from CG4, after repeated analyses with borderline results (Moser et al. 1995). PBD6-01 is the only patient whose CG assignment had remained in doubt until now. In an earlier publication (Moser et al. 1995), three patients were assigned to CG6. Subsequently, two of these patients were found to have mutations in PEX6 (Yahraus et al. 1996), and they were therefore reassigned to CG4. Earlier efforts to demonstrate a mutation in patient PBD6-01 had been unsuccessful. This, combined with the equivocal results of biochemical studies referred to earlier, had made 
it impossible to exclude the existence of CG6, prior to the new mutation analysis presented here. In this report, we provide several lines of novel evidence at the molecular level, indicating that CG6 is the same CG as CG4.

\section{Materials and methods}

\section{Cell lines}

Skin fibroblast cell lines from patients, including fibroblasts from a CG6 patient (PBD6-01) with NALD (Kelly et al. 1986), were cultured in Dulbecco's modified Eagle's medium-high glucose (DMEM) supplemented with 10\% fetal bovine serum, as described (Shimozawa et al. 1992). CHO cell mutants, including pex6 ZP92 (Shimozawa et al. 1992; Tsukamoto et al. 1995) and pex1 ZP107 (Tamura et al. 1998), were cultured as described.

\section{Cell fusion and DNA transfection}

Parent fibroblasts and cells to be fused were cocultured for 1 day and then fused using polyethylene glycol, as described (Shimozawa et al. 1992; Tsukamoto et al. 1990). Cells were stained using specific antibodies 3 days after cell fusion. DNA transfection was performed by electroporation, using a Gene Pulser II electroporator (Bio-Rad, Hercules, CA, USA) with the setting of $320 \mathrm{~V} / 500 \mu \mathrm{F}$ and by lipofection, using Lipofectamine (Life Technologies, Tokyo, Japan) as described (Tamura et al. 1998).

\section{Morphological analysis}

Peroxisomes in human fibroblasts and $\mathrm{CHO}$ cells were visualized by indirect immunofluorescence microscopy, as described (Shimozawa et al. 1992). Rabbit antibody to human catalase (Sigma, St. Louis, MO, USA) was raised by conventional subcutaneous injection. Antigen-antibody complexes were detected by fluorescein isothiocyanate (FITC)-labeled goat anti-rabbit immunoglobulin G ( $\mathrm{IgG})$ antibody (Cappel, Durham, NC, USA), under an Axioskop FL microscope (Carl Zeiss, Oberkochen, Germany).

\section{Isolation of $P E X 6$}

Human PEX6 cDNA was cloned by colony hybridization of a human liver cDNA library (Tamura et al. 1998), using as a probe a partial sequence of PEX6 (Fukuda et al. 1996) amplified by polymerase chain reaction (PCR) with a set of primers comprising nucleotide residues at 2375 2393 and 2913-2943. Subsequent sequencing of a cDNA clone was done by the dideoxy-chain termination method, using a Dye-terminator DNA sequence kit (Applied Biosystems, Foster City, CA, USA), as described (Tamura et al. 1998).

\section{Mutation analysis}

Poly $(\mathrm{A})^{+}$RNA was obtained from cultured patient's fibroblasts, using a QuickPrep Micro mRNA Purification kit (Amersham Pharmacia Biotech, Tokyo, Japan). Reverse transcription (RT)-PCR was done as described (Honsho et al. 1998), using poly(A) ${ }^{+}$RNA and a pair of HsPEX6specific PCR primers: a sense forward (F)1: 5'-GTCCTG TCACCATGGCGC-3' (initiation codon, underlined) and an antisense reverse (R)1: 5'-(CTGGGTCTAGA) CTA GCAGGCAGCAAACTTGCGCTGGATGCGC-3'

( $X b a \mathrm{I}$ site, in parenthesis; termination codon, underlined), containing nucleotide residues at positions -11 to 7 (A starting in the initiation codon as 1) and 2913-2943, to cover a full length of the PEX6 open reading frame. Amplification was done using the RT product as a template, with a forward primer $\mathrm{F} 1$ and a reverse XN.R: 5'GCTAGCTG(ATGCAT)GTTCACCTCCTGGCCCAG$3^{\prime}$ (NsiI site, in parenthesis; residues at 1840-1871). Amplification was, likewise, performed by the use of a set of primers: a forward F4, 5' -AGATGGACCAGCCAGTGC$3^{\prime}$ (residues at 1179-1196), and a reverse R1, as well as another set: a forward F2-1, 5'-GGTTGGGCACTG CTTGGCAC-3' (residues at 310-329), and R2, 5'GCCAAAGAGTCCAGTTCATC-3' (residues at 24072426). Respective cDNA clones were cloned into pGEM-T Easy vector (Promega, Madison, WI, USA) and were sequenced.

Genomic DNA was prepared from cultured fibroblasts, as described (Laird et al. 1991). To determine the zygosity of the mutant PEX6 alleles (see Fig. 2) in patient PBD601 , PCR was performed to amplify the sequence encompassing nucleotide residues $619-882$ in the human PEX6 gene (GenBank accession number, AF108095-AF108098) (Zhang et al. 1999), using a pair of PEX6-specific primers, a forward F3-1: 5'-CTTTTAATCTTGGCTGTGAC-3' (nucleotide residues at 833-852 in exon 1) and a reverse 42.R: 5'-CCGTCATAATTTCCATTAGTG-3' (residues at 996-1016 in exon 2), and another set of primers, a forward F2: 5'-GGGCTCGGACCGCGAGTC-3' (residues at 343360 in exon 1) and a reverse IVS1-R: $5^{\prime}$-CGGCTAGAA ACATTATGTTCA-3' (residues at 109-129 in intron 1). Next, to amplify the sequence between nucleotide residues at 2095-2362, PCR was, likewise, done using a set of primers, a forward F6: 5'-TGGCAGGTGGCTTGACTG-3' (nucleotide residues at 1964-1981 in exon 10) and a reverse R2 (see above). PCR products were cloned into pGEM-T Easy vector and sequenced.

\section{Generation of mutant constructs}

An expression vector carrying the patient PBD6-01-derived $P E X 6$ was constructed as follows. PCMVSPORT $\cdot H s P E X 6$ was first digested with $X b a \mathrm{I}$ and HindIII, blunted, and then ligated. A 264-bp deleted form of PEX6, termed PEX6del619-882 (see Results), in pCMVSPORT was formed by replacing a BssHII-XhoI fragment of the pCMVSPORT $H s P E X 6$ with a BssHII-XhoI fragment of 
the patient PBD6-01-derived PEX6del619-882 in pGEM-T Easy vector. The other mutant type, termed PEX6del20952362/21ins, with a 268-bp deletion at positions from 2095 to 2362, plus a following 21-bp insertion, was constructed by replacing a XhoI-NotI fragment of the pCMVSPORT $\cdot H s P E X 6$ with a XhoI-NotI fragment of the patient-derived PEX6del2095-2362/21 ins in pGEM-T Easy vector. Both constructs were confirmed by nucleotide sequence analysis.

\section{Results and discussion}

\section{CG analysis}

In the present work, as a step toward searching for a complementing cDNA for CG6, we first re-evaluated CG analysis of PBD6-01 fibroblasts, basically by cell fusion and $P E X$ gene transfection. In the patient's fibroblasts, peroxisomes were not detected when the cells were immunostained with an anti-catalase antibody (Fig. 1a). Peroxisome-positive cells were occasionally discernible by cell staining, using antibody to peroxisomal targeting signal type 1 (called PTS1) (data not shown). We performed cell fusion between CG6 PBD6-01 fibroblasts and fibroblasts from a $P E X 6$-defective CG4 patient (PBDC-03) with ZS (Fukuda et al. 1996), as well as a PEX1-defective CG1 ZS patient (PBDE-14) (Imamura et al. 1998). Numerous peroxisomes were detected 3 days after the fusion of PBD6-01 fibroblasts with CG1 cells, as assessed by staining with anticatalase antibody (Fig. 1d), whereas no peroxisome was visible in cells fused with CG4 fibroblasts (Fig. 1b). Moreover, cell fusion of PBD6-01 fibroblasts with a $\mathrm{CHO}$ pex6 mutant ZP92 did not restore peroxisomal protein import, while a cell hybrid with a pex1 ZP107 showed numerous catalase-positive structures, i.e., peroxisomes (Fig. 1, $c$ and
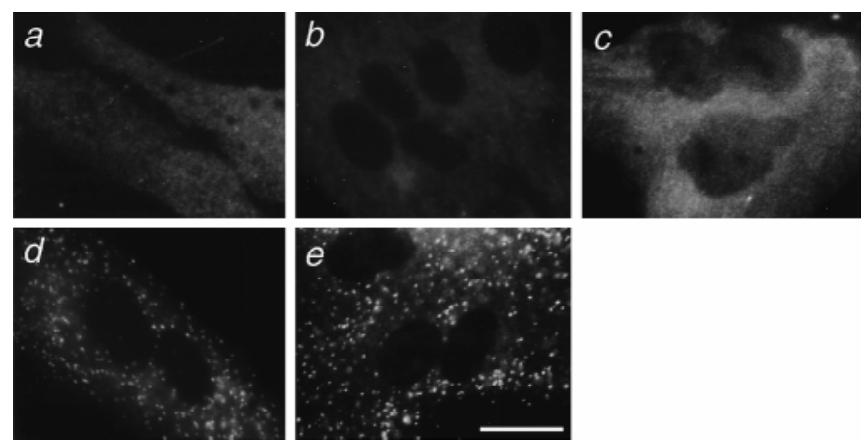

Fig. 1. Cell fusion analysis. Fibroblasts from a complementation group (CG)6 neonatal adrenoleukodystrophy (NALD) patient (PBD6-01) were fused with peroxisome-deficient cells of other CGs. a, CG6 PBD6-01 fibroblasts; $b$ and $c$, cell fusion was done with fibroblasts from a PEX6-defective CG4 Zellweger syndrome (ZS) patient and Chinese hamster ovary (CHO) pex6 mutant cells, respectively; $d$ and $e$, PBD601 fibroblasts fused with $P E X 1$-deficient CG1 patient's fibroblasts and CHO pex1 mutant ZP107, respectively. Cells were stained with antihuman catalase antibody. Note that peroxisomes were restored in $d$ and $e$, but not in $b$ and $c$. Bar $30 \mu \mathrm{m}$ e). These observations suggested that CG6 is the same CG as $P E X 6$-defective CG4. The formation of peroxisomes was, likewise, observed after cell fusion between PBD6-01 fibroblasts and fibroblasts from the other 10CGs of PBDs (data not shown). We have observed that PBD6-01 fibroblasts are unstable with respect to peroxisome deficiency, perhaps representing a mosaicism of peroxisomes in this NALD patient, and having led us to the previous conclusion, i.e., assignment of PBD6-01 to CG6. It is of interest to note that the very early development of the patient PBD6-01 was not considered abnormal (Kelly et al. 1986), suggesting that, in vivo, the peroxisomal protein import deficit was not complete.

We cloned human PEX6 cDNA, encoding a 980-aminoacid AAA ATPase family peroxin, by colony hybridization of a human liver cDNA library (Tamura et al. 1998). Sequencing of one full-length cDNA out of several clones showed a single base-change in a codon for Pro ${ }^{939}$ (CCA) (Fukuda et al. 1996; Yahraus et al. 1996) to $\operatorname{Gln}^{939}$ (CAA), implying a single nucleotide polymorphism in PEX6 (GenBank accession number, AB051076). We then introduced this $P E X 6$ cDNA into PBD CG6 fibroblasts by electroporation. Numerous peroxisomes were detected 3 days post-transfection (Fig. 2A, a). Expression of Pex6p possessing Pro $^{939}$, likewise, complemented peroxisome biogenesis (data not shown). PBD6 fibroblasts were also transfected with human PEX1 cDNA (Tamura et al. 1998) encoding another AAA-family peroxin with a mass of $143 \mathrm{kDa}$. Peroxisomes were not evident in the transfectants (data not shown). Furthermore, no complementation was evident upon transfection of PBD6 fibroblasts with other human $P E X$ cDNAs (data not shown). Therefore, $P E X 6$ was most likely to be impaired in CG6 PBDs.

\section{Mutation analysis}

To determine the dysfunction of PEX6 in PBD6-01 fibroblasts, we performed reverse transcription (RT)-PCR, using poly $(\mathrm{A})^{+}$RNA and a pair of HsPEX6-specific PCR primers: a sense F1 and an antisense R1. Amplification was done using the RT product as a template with a forward primer F1 and a reverse XN.R. Amplification was, likewise, performed by the use of a set of primers: a forward F4 and a reverse R1, as well as another set: a forward F2-1 and R2. Subsequent sequencing of the respective cDNA clones revealed that 7 of 12 clones had a 264-bp deletion of nucleotide residues at positions from 619 to 882 , termed PEX6del619-882, resulting in an 88-amino-acid truncation in one allele (Fig. 2B, upper panel) (GenBank accession number, AB051077). Of note, these clones showed Pro ${ }^{939}$ (see above). The truncated sequence corresponded to about one-third of the $3^{\prime}$-side of exon 1 (Zhang et al. 1999). Another type of mutation was identified in 6 of 10 clones: a 268-bp deletion at positions from 2095 to 2362, plus a following 21-bp insertion, termed PEX6del2095-2362/21ins, resulting in the amino-acid sequence from the $\mathrm{N}$-terminus to the residue at 698 of the normal Pex6p, followed by a distinct 40-amino-acid sequence and a termination (Fig. 2B, 
A

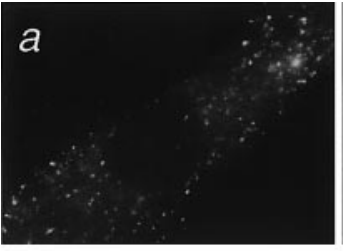

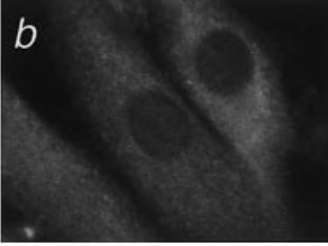

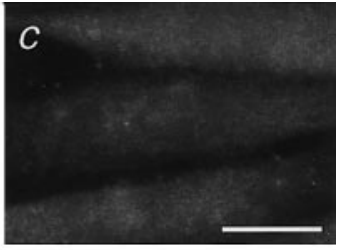

B

Control

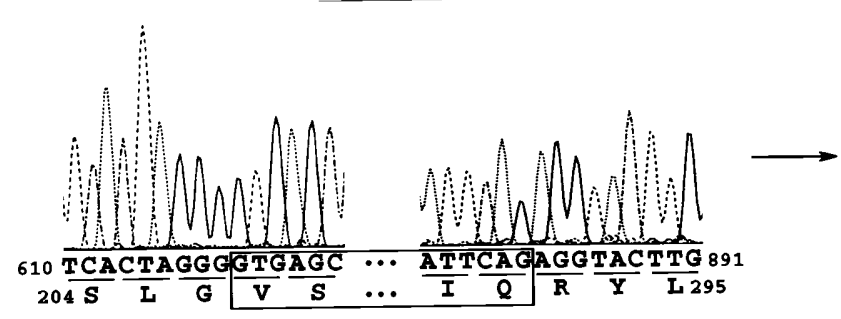

Control

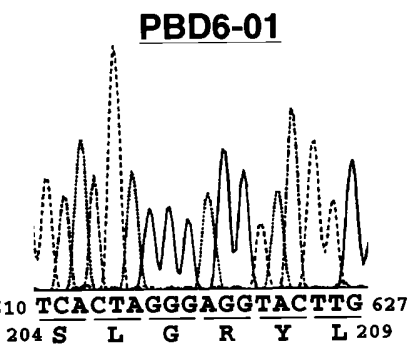

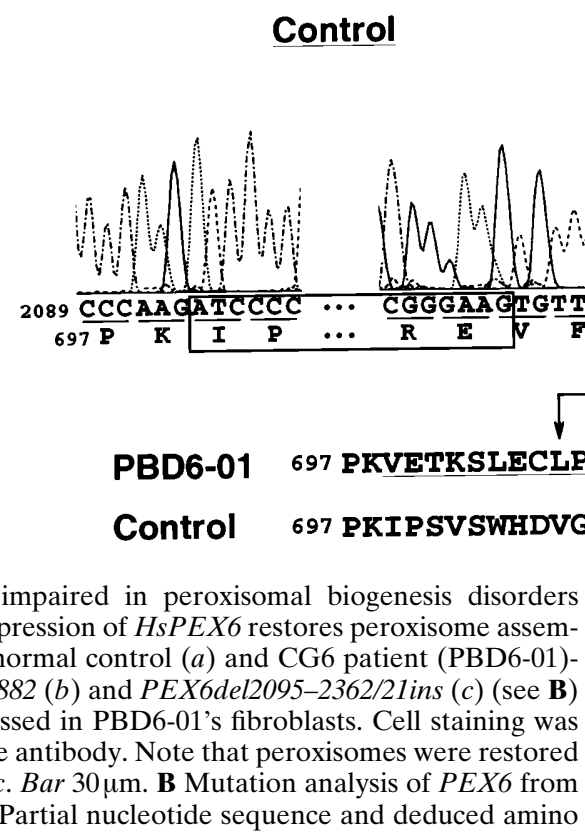

lower panel) (GenBank accession number, AB051078). These mutated forms of PEX6 possessed $\mathrm{Gln}^{939}$ (see above). The deleted sequence 2095 to 2362 encompasses exons 11 and 12 (Zhang et al. 1999). These mutations inactivated the function of $P E X 6$, as verified by the expres-sion of PEX6del619-882 and PEX6del2095-2362/ 21ins in PBD6-01 fibroblasts (Fig. 2A, $b$ and $c$ ), as well as in $\mathrm{CHO}$ pex6 ZP92 cells (data not shown), where peroxisomes were not re-established. The data collectively indicate that mutation of PEX6 is the genetic-related cause of CG6 PBDs.

To confirm the mutation sites and zygosity of the mutant alleles, genomic PCR was done to amplify the sequence encompassing nucleotide residues 619-882 in the human PEX6 gene (Zhang et al. 1999). In 8 of 11 independent clones isolated from PCR products in the search for a deletion of residues at 619-882, we identified a single nucleotide substitution at $399, \mathrm{~T} \rightarrow \mathrm{G}$ (a silent mutation in a codon for acid sequence of PEX6 cDNA isolated from a normal control (left panel) and the patient PBD6-01 (right panel) are shown. A 264-bp deletion of nucleotide residues from 619 to 882 (upper panel, boxed), termed PEX6del619-882, and a 268-bp deletion from 2095-2362, plus a 21-bp insertion (lower panel) inducing a 40-amino acid sequence entirely distinct from normal Pex6p (bottom, underlined), termed PEX6del2095-2362/21ins, were identified

${ }^{133} \mathrm{~V}$ ), and a 14-bp deletion at 800-813 in exon 1, comprising residues $1-882$, which, presumably, gave rise to the deletion of residues 619-882 (data not shown). Three of the 11 clones showed the normal PEX6 sequence. Next, to amplify the sequence between nucleotide residues at 2095-2362, PCR was, likewise, done. In 5 of 6 independent clones, a point mutation, $\mathrm{G} \rightarrow \mathrm{A}$, at the $3^{\prime}$-terminus of exon 12 was identified, which apparently caused a deletion of exons 11 and 12. One of the 6 clones contained the normal PEX6 gene sequence. We interpreted these results to mean that this NALD patient, PBD6-01, was a compound heterozygote for the mutations. Each of the two alleles, the PEX6del619-882 allele, apparently encoding for the Pro ${ }^{939}$-Pex6p truncated at 207-294, and the other ellele, PEX6del2095-2362/21ins, apparently encoding for the $\mathrm{Gln}^{939}$-Pex6p with a deletion in the C-terminal part encoded by exons 11 and 12 plus a frameshift/termination, was, most likely, derived from each parent. 
Table 1. Complementation groups of patients with PBDs, and of $\mathrm{CHO}$ cell mutants

CG Designation of

patients with PBDs

\begin{tabular}{|c|c|c|c|c|}
\hline Japanese & $\begin{array}{l}\text { US/ } \\
\text { European }\end{array}$ & Phenotype & $\begin{array}{l}\mathrm{CHO} \\
\text { mutant }^{\mathrm{a}}\end{array}$ & Gene \\
\hline A & 8 & ZS, NALD, IRD & ZP124 & \\
\hline B & $7(5)^{\mathrm{b}}$ & ZS, NALD & & PEX10 \\
\hline $\mathrm{C}$ & $4(6)$ & ZS, NALD & ZP92 & PEX6 \\
\hline $\mathrm{D}$ & 9 & $\mathrm{ZS}$ & & PEX16 \\
\hline $\mathrm{E}$ & 1 & ZS, NALD, IRD & Z24/ZP107 & $P E X 1$ \\
\hline $\mathrm{F}$ & 10 & ZS, IRD & Z65 & $P E X 2$ \\
\hline $\mathrm{G}$ & $12^{\mathrm{c}}$ & $\mathrm{ZS}$ & ZPG208 & $P E X 3$ \\
\hline $\mathrm{H}$ & $13^{\mathrm{c}}$ & ZS, NALD & ZP128 & PEX13 \\
\hline \multirow[t]{3}{*}{$\mathrm{J}$} & $14^{\mathrm{c}}$ & $\mathrm{ZS}$ & ZP119 & PEX19 \\
\hline & 2 & ZS, NALD & ZP105/ZP139 & PEX5 \\
\hline & 3 & ZS, NALD, IRD & ZP109 & PEX12 \\
\hline \multirow[t]{4}{*}{$\mathrm{R}$} & 11 & RCDP & ZPG207 & $P E X 7$ \\
\hline & & & ZP110 & PEX14 \\
\hline & & & ZP114 & \\
\hline & & & ZP126 & \\
\hline
\end{tabular}

Numbers in parentheses represent the previously assigned, distinct CGs

PBD, Peroxisomal biogenesis disorders; $\mathrm{CHO}$, Chinese hamster ovary; CG, complementation groups; ZS, Zellweger syndrome; NALD, neonatal adrenoleukodystrophy; IRD, infantile Refsum disease; RCDP, rhizomelic chondrodysplasia punctata

${ }^{a}$ From (Fujiki 2000; Ghaedi et al. 2000)

${ }^{\mathrm{b}}$ From (Moser et al. 1995; Shimozawa et al. 1998)

${ }^{\mathrm{c}}$ From (Gould and Valle 2000)

Accordingly, by merging CG6 with CG4, human PBDs are now classified into $11 \mathrm{CGs}$ of peroxisome-deficient PBDs such as ZS, NALD, and IRD, and one CG of PEX7defective RCDP, impaired solely in the PTS2 import (Table 1).

Acknowledgments We thank other members of the Fujiki laboratory for discussions. This work was supported in part by a CREST grant (to Y.F.) from the Japan Science and Technology Corporation; and by Grants-in-Aid for Scientific Research (to Y.F.) from The Ministry of Education, Science, Sports, and Culture of Japan.

\section{References}

Fujiki Y (2000) Peroxisome biogenesis and peroxisome biogenesis disorders. FEBS Lett 476:42-46

Fukuda S, Shimozawa N, Suzuki Y, Zhang Z, Tomatsu S, Tsukamoto T, Hashiguchi N, Osumi T, Masuno M, Imaizumi K, Kuroki Y, Fujiki Y, Orii T, Kondo N (1996) Human peroxisome assembly factor-2 (PAF-2): a gene responsible for group $\mathrm{C}$ peroxisome biogenesis disorder in humans. Am J Hum Genet 59:1210-1220

Ghaedi K, Honsho M, Shimozawa N, Suzuki Y, Kondo N, Fujiki Y (2000) PEX3 is the causal gene responsible for peroxisome membrane assembly-defective Zellweger syndrome of complementation group G. Am J Hum Genet 67:976-981

Ghaedi K, Kawai A, Okumoto K, Tamura S, Shimozawa N, Suzuki Y, Kondo N, Fujiki Y (1999) Isolation and characterization of novel peroxisome biogenesis-defective Chinese hamster ovary cell mutants using green fluorescent protein. Exp Cell Res 248:489497

Gould SJ, Valle D (2000) Peroxisome biogenesis disorders: genetics and cell biology. Trends Genet 16:340-345

Honsho M, Tamura S, Shimozawa N, Suzuki Y, Kondo N, Fujiki Y (1998) Mutation in PEX16 is causal in the peroxisome-deficient Zellweger syndrome of complementation group D. Am J Hum Genet 63:1622-1630

Imamura A, Tamura S, Shimozawa N, Suzuki Y, Zhang Z, Tsukamoto T, Orii T, Kondo N, Osumi T, Fujiki, Y (1998) Temperaturesensitive mutation in PEX1 moderates the phenotypes of peroxisome deficiency disorders. Hum Mol Genet 7:2089-2094

Kelly RI, Datta NS, Dobyns WB, Hajra AK, Moser AB, Noetzel MJ, Zackai EH, Moser HW (1986) Neonatal adrenoleukodystrophy: new cases, biochemical studies, and differentiation from Zellweger and related peroxisomal polydystrophy syndromes. Am J Med Genet 23:869-901

Laird PW, Zijderveld A, Linders K, Rudnicki MA, Jaenisch R, Berns A (1991) Simplified mammalian DNA isolation procedure. Nucleic Acids Res 19:4293

Lazarow PB, Moser HW (1995) Disorders of peroxisome biogenesis. In: Scriver CR, Beaudet AI, Sly WS, Valle D (eds) The metabolic basis of inherited disease. McGraw-Hill, New York, pp 2287-2324

Moser AB, Rasmussen M, Naidu S, Watkins PA, McGuiness M, Hajra AK, Chen G, Raymond G, Liu A, Gordon D, Garnaas K, Walton DS, Skjeldal OH, Guggenheim MA, Jackson LG, Elias ER, Moser HW (1995) Phenotype of patients with peroxisomal disorders subdivided into 16 complementation groups. J Pediatr 127:13-22

Muntau AC, Mayerhofer PU, Paton BC, Kammerer S, Roscher AA (2000) Defective peroxisome membrane synthesis due to mutations in human $P E X 3$ causes Zellweger syndrome, complementation group G. Am J Hum Genet 67:967-975

Shimozawa N, Suzuki Y, Zhang Z, Imamura A, Ghaedi K, Fujiki Y, Kondo N (2000) Identification of PEX3 as the gene mutated in a Zellweger syndrome patient lacking peroxisomal remnant structures. Hum Mol Genet 9:1995-1999

Shimozawa N, Suzuki Y, Zhang Z, Imamura A, Kondo N, Kinoshita N, Fujiki Y, Tsukamoto T, Osumi T, Imanaka T, Orii T, Beemer F, Mooijer P, Dekker C, Wanders RJA (1998) Genetic basis of peroxisome-assembly mutants of humans, Chinese hamster ovary cells and yeast: identification of a new complementation group of peroxisomebiogenesis disorders apparently lacking peroxisomal-membrane ghosts. Am J Hum Genet 63:1898-1903

Shimozawa N, Tsukamoto T, Suzuki Y, Orii T, Fujiki Y (1992) Animal cell mutants represent two complementation groups of peroxisomedefective Zellweger syndrome. J Clin Invest 90:1864-1870

South ST, Sacksteder KA, Li X, Liu Y, Gould SJ (2000) Inhibitors of COPI and COPII do not block PEX3-mediated peroxisome synthe sis. J Cell Biol 149:1345-1360

Tamura S, Okumoto K, Toyama R, Shimozawa N, Tsukamoto T, Suzuki Y, Osumi T, Kondo N, Fujiki Y (1998) Human PEX1 cloned by functional complementation on a $\mathrm{CHO}$ cell mutant is responsible for peroxisome-deficient Zellweger syndrome of complementation group I. Proc Natl Acad Sci USA 95:4350-4355

Tsukamoto T, Miura S, Nakai T, Yokota S, Shimozawa N, Suzuki Y, Orii T, Fujiki Y, Sakai F, Bogaki A, Yasumo H, Osumi T (1995) Peroxisome assembly factor-2, a putative ATPase cloned by functional complementation on a peroxisome-deficient mammalian cell mutant. Nature Genet 11:395-401

Tsukamoto T, Yokota S, Fujiki Y (1990) Isolation and characterization of Chinese hamster ovary cell mutants defective in assembly of peroxisomes. J Cell Biol 110:651-660

Yahraus T, Braverman N, Dodt G, Kalish JE, Morrell JC, Moser HW, Valle D, Gould SJ (1996) The peroxisome biogenesis disorder group 4 gene, $P X A A A 1$, encodes a cytoplasmic ATPase required for stability of the PTS1 receptor. EMBO J 15:2914-2923

Zhang Z, Suzuki Y, Shimozawa N, Fukuda S, Imamura A, Tsukamoto T, Osumi T, Fujiki Y, Orii T, Wanders RJA, Barth PG, Moser HW, Paton BC, Besley GT, Kondo N (1999) Geneomic structure and identification of 11 novel mutations of PEX6 (peroxisome assembly factor-2) gene in patients with peroxisome biogenesis disorders. Hum Mut 13:487-496 